\title{
Superconvergence for Multistep Collocation
}

\author{
By Ivar Lie and Syvert P. Nørsett
}

\begin{abstract}
One-step collocation methods are known to be a subclass of implicit RungeKutta methods. Further, one-leg methods are special multistep one-point collocation methods. In this paper we extend both of these collocation ideas to multistep collocation methods with $k$ previous meshpoints and $m$ collocation points. By construction, the order is at least $m+k-1$. However, by choosing the collocation points in the right way, order $2 m+k-1$ is obtained as the maximum. There are $\left(\begin{array}{c}m+k-1 \\ k-1\end{array}\right)$ sets of such "multistep Gaussian" collocation points.
\end{abstract}

1. Introduction. We study the numerical solution of the initial value problem

$$
y^{\prime}(x)=f(x, y), \quad y\left(x_{0}\right)=y_{0} .
$$

One-step collocation methods for initial value problems have been investigated thoroughly; see, e.g., [13], [16], [14] and [6]. These methods are a subclass of implicit Runge-Kutta methods

$$
\begin{gathered}
Y_{i}=y_{n}+h \sum_{j=1}^{m} a_{i j} Y_{j}, \quad i=1, \ldots, m ; \\
y_{n+1}=y_{n}+h \sum_{j=1}^{m} b_{j} f\left(Y_{j}\right),
\end{gathered}
$$

where $h$ is the stepsize.

The order is at most $2 m$ and is attained for Gaussian collocation points. In that case, the implementation is rather costly, which is related to the fact that the Runge-Kutta matrix $A=\left\{a_{i j}\right\}_{i, j=1}^{m}$ has at least $m-1$ complex eigenvalues. The optimal case is a one-point spectrum matrix $A$. Methods of that type are given in [11], where the collocation points are related to zeros of Laguerre polynomials. However, the order of such singly implicit methods is restricted to $m+1$.

The idea behind multistep collocation is to let the collocation polynomial use information from previous points in the integration. This is illustrated in the figure below for 3 previous points and 2 collocation points.

In this paper we study methods with Lagrange type interpolation conditions (see also [9]). Methods with Hermite type conditions are discussed in [8].

Multistep collocation methods are a subclass of the General Linear Methods of Butcher, and we will prove that this subclass is actually a subclass of multistep Runge-Kutta methods. Important special cases of multistep collocation are the one-leg methods of Dahlquist [4], [5] and the BDF-methods. They are obtained using one collocation point and $k$ interpolation points.

Received October 20, 1986; revised October 7, 1987.

1980 Mathematics Subject Classification (1985 Revision). Primary 65L05. 


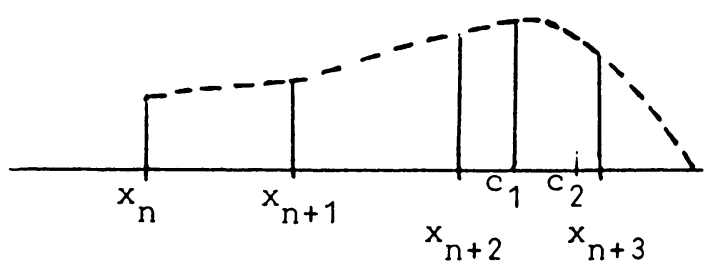

FIGURE 1

Multistep collocation polynomial.

As to the order, we prove that the maximum attainable order is $2 m+k-1$. The proof will use the Alekseev-Gröbner theorem and a specific collocation style technique. This family of superconvergent methods is related to Gaussian quadrature, and we list some of these "multistep Gauss points".

In a later paper we will return to other aspects of multistep collocation: $C$ polynomial theory, the question of singly-implicitness, analysis for variable stepsize, error estimation and stability properties.

2. Multistep Collocation. In this section we consider the construction of multistep collocation methods for constant stepsize $h$ and give expressions for the coefficients for arbitrary values of the number of steps and the number of collocation points. These expressions will be seen to be natural generalizations of the corresponding coefficients in the one-step case. Let $u_{n+i}$ be approximations to $y_{n+i}=y\left(x_{n+i}\right), i=0,1, \ldots, k-1$, and $c_{1}, \ldots, c_{m}$ distinct real numbers. Then a $k$-step multistep collocation method with $m$ collocation points is constructed as follows:

Find $u \in \Pi_{m+k-1}, k>0, m>0$, such that

$$
\begin{gathered}
u\left(x_{n+i}\right)=u_{n+i}, \quad i=0,1, \ldots, k-1 \\
u^{\prime}\left(x_{n+k-1}+c_{j} h\right)=f\left(x_{n+k-1}+c_{j} h, u\left(x_{n+k-1}+c_{j} h\right)\right), \quad j=1, \ldots, m .
\end{gathered}
$$

Then as an approximation to $y_{n+k}$ we take

$$
u_{n+k}=u\left(x_{n+k}\right) \text {. }
$$

In order to characterize multistep collocation as multistep Runge-Kutta methods; we state the following lemma.

LEMMA 1. The multistep collocation method (2.1), (2.2), (2.3) can be written as a multistep Runge-Kutta method,

$$
\begin{aligned}
Y_{j}= & \sum_{i=0}^{k-1} \phi_{i}\left(t_{n+k-1}+c_{j} h\right) y_{n+k-1-i} \\
& +h \sum_{i=1}^{m} \psi_{i}\left(t_{n+k-1}+c_{j} h\right) f\left(t_{n+k-1}+c_{i} h, Y_{i}\right), \quad j=1, \ldots, m \\
Y_{n+k} & =\sum_{i=0}^{k-1} \phi_{i}\left(t_{n+k}\right) y_{n+k-1-i}+h \sum_{i=1}^{m} \psi_{i}\left(t_{n+k}\right) f\left(t_{n+k-1}+c_{i} h, Y_{i}\right)
\end{aligned}
$$


where $\phi_{i}(s), \psi_{i}(s) \in \Pi_{k+m-1}$ are uniquely given by

$$
\psi_{i}(s)=\frac{\tilde{D}_{k, i}}{D_{k-1}}, \quad k \geq 1, D_{0}=1,1 \leq i \leq m,
$$

with

$$
D_{k-1}=\operatorname{det}\left\{\int_{-i}^{0} \tau^{j-1} M(\tau) d \tau\right\}_{i, j=1}^{k-1}
$$

and

$$
\tilde{D}_{k, i}=\operatorname{det}\left[\begin{array}{llll}
\int_{0}^{s} l_{i}(s) d s & \int_{0}^{s} M(s) d s & \cdots & \int_{0}^{s} s^{k-2} M(s) d s \\
\int_{-1}^{0} l_{i}(s) d s & \int_{-1}^{0} M(s) d s & \cdots & \int_{-1}^{0} s^{k-2} M(s) d s \\
\vdots & \vdots & & \vdots \\
\int_{-(k-1)}^{0} l_{i}(s) d s & \int_{-(k-1)}^{0} M(s) d s & \cdots & \int_{-(k-1)}^{0} s^{k-2} M(s) d s
\end{array}\right],
$$

where

$$
M(t)=\prod_{i=1}^{m}\left(t-c_{i}\right), \quad l_{i}(t)=\frac{M(t)}{\left(t-c_{i}\right) M^{\prime}\left(c_{i}\right)}
$$

Further,

$$
\begin{gathered}
\phi_{0}(s)=\frac{D_{k, e}}{D_{k-1}}, \quad k \geq 1, D_{0}=1 ; \\
\phi_{i}(s)=\frac{D_{k}^{i}}{D_{k-1}}, \quad k \geq 1, D_{0}=1, \quad 1 \leq i \leq k-1,
\end{gathered}
$$

with

$$
D_{k, e}=\operatorname{det}\left[\begin{array}{cllll}
1 & \int_{0}^{s} M(s) d s & \int_{0}^{s} s M(s) d s & \cdots & \int_{0}^{s} s^{k-2} M(s) d s \\
-1 & \int_{-1}^{0} M(s) d s & \int_{-1}^{0} s M(s) d s & \cdots & \int_{-1}^{0} s^{k-2} M(s) d s \\
\vdots & \vdots & & & \vdots \\
-1 & \int_{-(k-1)}^{0} M(s) d s & \int_{-(k-1)}^{0} s M(s) d s & \cdots & \int_{-(k-1)}^{0} s^{k-2} M(s) d s
\end{array}\right]
$$

and

$$
D_{k}^{i}=\operatorname{det}\left[\begin{array}{clll}
0 & \int_{0}^{s} M(s) d s & \cdots & \int_{0}^{s} s^{k-2} M(s) d s \\
\vdots & & & \\
0 & & \cdots & \int_{-1}^{0} s^{k-2} M(s) d s \\
1 & \int_{-1}^{0} M(s) d s & & \\
0 & & & \\
\vdots & & & \\
0 & \int_{-(k-1)}^{0} M(s) d s & \cdots & \int_{-(k-1)}^{0} s^{k-2} M(s) d s
\end{array}\right]
$$

where the one in the first column is in position $i+1$.

Proof. To see that multistep collocation are multistep Runge-Kutta methods, put $Y_{j}=u\left(t_{n+k-1}+c_{j} h\right), k_{j}=u^{\prime}\left(t_{n+k-1}+c_{j} h\right)=f\left(t_{n+k-1}+c_{j} h, Y_{j}\right)$. Consider 
the interpolation problem (2.1), (2.2) for $u$. The interpolation data are $u_{n+i}, i=$ $0, \ldots, k-1$, and $f\left(t_{n+k-1}+c_{j} h, Y_{j}\right), j=1, \ldots, m$. The interpolation polynomial can be written in the following form:

$$
u(t)=\sum_{i=0}^{k-1} \phi_{i}(t) u_{n+k-1-i}+h \sum_{i=1}^{m} \psi_{i}(t) f\left(t_{n+k-1}+c_{i} h, Y_{i}\right) .
$$

(2.4) and (2.5) then follow from (2.13) and (2.3).

We now exhibit the form of the coefficients. We will use the scaled time variable $s=\left(t-t_{n+k-1}\right) / h$. This implies, e.g., that (2.4) can be written as

$$
Y_{j}=\sum_{i=0}^{k-1} \phi_{i}\left(c_{j}\right) y_{n+k-1-i}+h \sum_{i=1}^{m} \psi_{i}\left(c_{j}\right) f\left(c_{i}, Y_{i}\right), \quad j=1, \ldots, m .
$$

Consider $\psi_{i}(s)$ first.

From the interpolation conditions (2.1), (2.2) and the expression for $u(t)$ in (2.13) we see that the following conditions are imposed on $\psi_{i}$ :

$$
\begin{aligned}
\psi_{i}(-r)=0, \quad r & =0,1, \ldots, k-1 ; \\
\psi_{i}^{\prime}\left(c_{j}\right) & =\delta_{i j} .
\end{aligned}
$$

The latter condition can be satisfied with a polynomial of the following form:

$$
\psi_{i}^{\prime}(s)=l_{i}(s)\left[1+\sum_{j=0}^{k-2} \alpha_{j}\left(s-c_{i}\right) s^{j}\right]=l_{i}(s)+\sum_{j=0}^{k-2} \frac{\alpha_{j}}{a_{i}} M(s) s^{j}
$$

where $a_{i}$ is the denominator of $l_{i}(s)$. Setting $\bar{\alpha}_{j}=\alpha_{j} / a_{i}$ and integrating, we find

$$
\psi_{i}(s)=\int_{0}^{s} l_{i}(\tau) d \tau+\sum_{j=0}^{k-2} \bar{\alpha}_{j} \int_{0}^{s} \tau^{j} M(\tau) d \tau
$$

and we have a linear system for the $\bar{\alpha}_{j}$ 's from (2.14) (excluding the trivial $\left.\psi_{i}(0)=0\right)$ :

or

$$
\begin{aligned}
& \psi_{i}(-1)=0, \\
& \quad \vdots \\
& \psi_{i}(-(k-1))=0,
\end{aligned}
$$

$$
\sum_{j=0}^{k-2} \bar{\alpha}_{j} \int_{-r}^{0} s^{j} M(s) d s=-\int_{-r}^{0} l_{i}(s) d s, \quad r=1, \ldots, k-1 .
$$

The determinant of the coefficient matrix of this system is $D_{k-1}$. By Cramer's rule we obtain

$$
\bar{\alpha}_{j}=D_{k-1, j} / D_{k-1},
$$

where $D_{k-1, j}$ is the determinant obtained by replacing the $j$ th column with the right-hand side of (2.17).

For $\psi_{i}(s)$ we have

$$
\psi_{i}(s)=\frac{1}{D_{k-1}}\left(\int_{0}^{s} l_{i}(s) d s D_{k-1}+\sum_{j=0}^{k-2} D_{k-1, j} \int_{0}^{s} s^{j} M(s) d s\right) .
$$


But the parenthesis above is just an expansion of $\tilde{D}_{k, i}$ along the first row. Thus, (2.6) is established.

Now consider $\phi_{i}(s)$. The conditions imposed on $\phi_{i}$ are

$$
\begin{gathered}
\phi_{i}^{\prime}\left(c_{j}\right)=0, \quad j=1, \ldots, m, i=0, \ldots, k-1 \\
\phi_{i}(-j)=\delta_{i j}, \quad i, j=0,1, \ldots, k-1 .
\end{gathered}
$$

The first part of the conditions can be fulfilled with

$$
\phi_{i}(s)=\alpha_{0}^{(i)}+\sum_{j=0}^{k-2} a_{j+1}^{(i)} \int_{0}^{s} s^{j} M(s) d s .
$$

Consider $\phi_{0}$ first. From $\phi_{0}(0)=1$ there follows that $\alpha_{0}^{(0)}=1$, and (2.20) gives

$$
\sum_{j=0}^{k-2} a_{j+1}^{(0)} \int_{-r}^{0} s^{j} M(s) d s=1, \quad r=1, \ldots, k-1 .
$$

This is identical to (2.17) except for the right-hand side. Thus we will have the same expression as (2.17) except for the first column of $\tilde{D}_{k-i}$ which will be substituted by all ones.

Now consider $\phi_{i}, i>0$. In this case, $a_{0}^{(i)}=0$, and (2.20) gives

$$
\sum_{j=0}^{k-2} a_{j+1}^{(i)} \int_{-r}^{0} s^{j} M(s) d s=-\delta_{r i}, \quad r=1, \ldots, k-1 .
$$

Thus we get the same expression as for $\phi_{0}(s)$ except for the first column of $D_{k, e}$ which is substituted by zero and $e_{i}$ (the $i$ th basis vector of $\mathbf{R}^{k-1}$ ).

Thus (2.10) is established.

Remark. We see that multistep collocation in the form (2.4), (2.5) can be put in the partitioned multivalue formalism of Burrage and Moss [3], which is given by

$$
\begin{aligned}
& Y_{i}=\sum_{j=1}^{k} a_{i j}^{(1)} y_{j}^{(n-1)}+h \sum_{j=1}^{m} b_{i j}^{(1)} f\left(t_{n+k-1}+c_{j} h, Y_{j}\right), \quad i=1, \ldots, m \\
& y_{i}^{(n)}=\sum_{j=1}^{k} a_{i j}^{(2)} y_{j}^{(n-1)}+h \sum_{j=1}^{m} b_{i j}^{(2)} f\left(t_{n+k-1}+c_{j} h, Y_{j}\right), \quad i=1, \ldots, k .
\end{aligned}
$$

Here, $Y_{i}, i=1, \ldots, m$, is a vector of interior ordinates and $y_{i}^{(n)}, i=1, \ldots, k$, is a vector of exterior ordinates in "generation" $n$. By setting

$$
\begin{array}{ll}
A_{1}=\left\{a_{i j}^{(1)}\right\} \in \mathbf{R}^{m \times k}, & B_{1}=\left\{b_{i j}^{(1)}\right\} \in \mathbf{R}^{m \times m} \\
A_{2}=\left\{a_{i j}^{(2)}\right\} \in \mathbf{R}^{k \times k}, & B_{2}=\left\{b_{i j}^{(2)}\right\} \in \mathbf{R}^{k \times m} \\
Y=\left[Y_{1}, \ldots, Y_{m}\right]^{T} \quad \text { and } & y^{(n)}=\left[y_{1}^{(n)}, \ldots, y_{k}^{(n)}\right]
\end{array}
$$

one can write more compactly

$$
\left[\begin{array}{c}
Y \\
y^{(n)}
\end{array}\right]=\left(\left[\begin{array}{ll}
A_{1} & B_{1} \\
A_{2} & B_{2}
\end{array}\right] \otimes I_{n}\right)\left[\begin{array}{l}
y^{(n-1)} \\
h f(Y)
\end{array}\right]
$$


Example 1. For $k=2$ the coefficients become

$$
\begin{gathered}
\psi_{i}(s)=\frac{1}{\int_{-1}^{0} M(s) d s}\left|\begin{array}{cc}
\int_{0}^{s} l_{i}(s) d s & \int_{0}^{s} M(s) d s \\
\int_{-1}^{0} l_{i}(s) d s & \int_{-1}^{0} M(s) d s
\end{array}\right| \\
=\int_{0}^{s} l_{i}(s)-\frac{\int_{-1}^{0} l_{i}(s) d s}{\int_{-1}^{0} M(s) d s} \int_{0}^{s} M(s) d s \\
\phi_{0}(s)=\frac{1}{\int_{-1}^{0} M(s) d s}\left|\begin{array}{cc}
1 & \int_{0}^{s} M(s) d s \\
-1 & \int_{-1}^{0} M(s) d s
\end{array}\right|=1+\frac{\int_{0}^{s} M(s) d s}{\int_{-1}^{0} M(s) d s}, \\
\phi_{1}(s)=\frac{1}{\int_{-1}^{0} M(s) d s}\left|\begin{array}{cc}
0 & \int_{0}^{s} M(s) d s \\
+1 & \int_{-1}^{0} M(s) d s
\end{array}\right|=-\frac{\int_{0}^{s} M(s) d s}{\int_{-1}^{0} M(s) d s}=1-\phi_{0}(s) .
\end{gathered}
$$

Two-step collocation is also studied in [12], but the general expressions for the coefficients are not given.

Example 2. We now give for $k=2, m=2$, a specific method with $c_{1}=1 / 4$, $c_{2}=1.0$. We get the following method:

$$
\begin{aligned}
& Y_{1}=\frac{475}{464} y_{n}-\frac{11}{464} y_{n-1}+h\left(\frac{85}{348} f\left(Y_{1}\right)-\frac{25}{132} f\left(Y_{2}\right)\right), \\
& Y_{2}=\frac{28}{29} y_{n}-\frac{1}{29} y_{n-1}+h\left(\frac{64}{87} f\left(Y_{1}\right)+\frac{26}{87} f\left(Y_{2}\right)\right)
\end{aligned}
$$

$$
y_{n+1}=Y_{2} \text {. }
$$

This method has order 3 and is almost $A(\alpha)$ stable with $\alpha$ close to $\pi / 2$.

3. Order Results. In this section we give order conditions for the multistep collocation method in two different forms, both of which are simpler than the corresponding conditions in "standard Runge-Kutta form" given by Burrage in [2]. This is possible because of the way multistep collocation methods are constructed. The order of multistep collocation is $m+k-1$ by construction, so assuming $m+$ $k-1>1$, the preconsistency and consistency conditions (see [6]) are automatically fulfilled.

3.1. Order Conditions of RK-Type. Burrage [2] considers the order of multistep RK-methods, and by referring to the matrices in the PMVM-formulation (Partitioned Multivalue Method, Burrage and Moss [3]), these conditions are as follows:

Let $c$ be the vector $\left[c_{1}, \ldots, c_{m}\right]^{T}$ and $q(t)$ be the term for the tree $t$ in the Butcher series for the starting procedure. The following short notation is introduced:

(3.1) $A(w): q(t)=q\left(\tau^{p}\right)$ for all rooted trees $\neq \tau^{p}$ of order $p$,

(3.2) $\bar{A}(w): A(w-1) \wedge\left(A_{2}-I_{k}\right)\left[q\left(\tau^{w}\right)-q(t)\right]=0$ for all $t \neq \tau^{w}$ of order $w$,

$$
\begin{aligned}
& B(w): p B_{2} c^{p-1}+\left(A_{2}-I_{k}\right) q\left(\tau^{p}\right)-\sum_{j=0}^{p-1}\left(\begin{array}{l}
p \\
j
\end{array}\right) q\left(\tau^{j}\right)=0, \quad p=0,1, \ldots, w, \\
& C(w): A_{1} q\left(\tau^{p}\right)=c^{p}-p B_{1} c^{p-1}, \quad p=0,1, \ldots, w .
\end{aligned}
$$


Then we have

$$
\bar{A}(w) \wedge B(w) \wedge C(w-1) \text { implies order } w .
$$

Burrage gives conditions for order up to $2 m+k-1$, but does not prove that solutions to the conditions really exist, notwithstanding a statement in [2] to this effect.

Instead of following Burrage's approach, we will in the following two paragraphs develop specific conditions for multistep collocation methods which are simpler than the ones above.

3.2. Superconvergence, Alekseev-Gröbner Approach. Consider now a multistep collocation with $k$ steps and $m$ stages as specified in (2.4) and (2.5). The corresponding collocation polynomial is given by

$$
M(s)=\prod_{i=1}^{m}\left(s-c_{j}\right) .
$$

In the one-step case, Nørsett and Wanner [13] showed that the Alekseev-Gröbner theorem gives a very short proof of order results. The same should be possible in the multistep case, and this is in fact what we use in the proof of the following theorem.

THEOREM 1. Let

$$
G_{i}=\operatorname{det}\left[\begin{array}{llc}
\int_{-1}^{0} M(s) s^{i} d s & \cdots & \int_{-1}^{0} M(s) s^{i+k-1} d s \\
\vdots & & \vdots \\
\int_{-(k-1)}^{0} M(s) s^{i} d s & \cdots & \int_{-(k-1)}^{0} M(s) s^{i+k-1} d s \\
\int_{0}^{1} M(s) s^{i} d x & \cdots & \int_{0}^{1} M(s) s^{i+k-1} d s
\end{array}\right] .
$$

Then the multistep collocation method (2.4), (2.5) has order $m+k-1+\nu$ if and only if $G_{i}=0$ for $i=0,1, \ldots, \nu-1$.

Proof. (We are grateful to G. Wanner for providing ideas to this proof.) We study the error in the method (2.1), (2.2) under the assumption that $u_{n+i}=y_{n+i}=$ $y\left(t_{n+i}\right), i=0, \ldots, k-1$, and use the Alekseev-Gröbner theorem (see, e.g., [12]):

$$
u(t)-y(t)=\int_{t_{n}}^{t} \Phi(t, \tau, u(\tau)) \cdot\left(u^{\prime}(\tau)-f(\tau, u(\tau))\right) d \tau
$$

where $\Phi$ is a variationai matrix. From the conditions (2.1) we get, using now the $s$-variable and assuming $h=1$ and $t_{n}=0$,

$$
\int_{-r}^{0} \Phi\left(u^{\prime}(\tau)-f(\tau, u(\tau))\right) d \tau=0, \quad r=0,1, \ldots, k-1 .
$$

With $p(\tau)=\Phi \cdot\left(u^{\prime}(\tau)-f(\tau, u(\tau))\right)$, the error is

$$
u(1)-y(1)=\int_{0}^{1} p(\tau) d \tau .
$$

We construct a quadrature formula similar to that in [13] for functions in the linear subspace

$$
V=\left\{p \mid \int_{-1}^{0} p(\tau) d \tau=0, \ldots, \int_{-(k-1)}^{0} p(\tau) d \tau=0\right\}
$$


Any $p \in V$ is approximated by an interpolation polynomial $\rho \in V$ such that $p\left(c_{i}\right)=$ $\rho\left(c_{i}\right)$ and hence

$$
p(s)-\rho(s)=M(s) \cdot r(s), \quad M(s) \cdot r(s) \in V .
$$

The error for the quadrature formula is then

$$
\int_{0}^{1} M(s) r(s) d s
$$

We want this error to be zero for $r$ a polynomial of as high a degree as possible. Let

$$
r_{i}(s)=\sum_{l=0}^{k-1} \alpha_{l} s^{i+l}, \quad i=0,1, \ldots
$$

Then the condition $M(s) r_{i}(s) \in V$ becomes

$$
\left[\begin{array}{llc}
\int_{-1}^{0} M(s) s^{i} d s & \cdots & \int_{-1}^{0} M(s) s^{i+k-1} d s \\
\vdots & & \vdots \\
\int_{-(k-1)}^{0} M(s) s^{i} d s & \cdots & \int_{-(k-1)}^{0} M(s) s^{i+k-1} d s
\end{array}\right] \cdot\left[\begin{array}{c}
\alpha_{0} \\
\vdots \\
\alpha_{k-1}
\end{array}\right]=0
$$

and the order condition $\int_{0}^{1} M(s) r_{i}(s) d s=0$ is

$$
\left[\int_{0}^{1} M(s) s^{i} d s, \ldots, \int_{0}^{1} M(s) s^{i+k-1} d s\right] \cdot\left[\begin{array}{c}
\alpha_{0} \\
\vdots \\
\alpha_{k-1}
\end{array}\right]=0 .
$$

(3.11) and (3.12) possess a nontrivial solution if and only if $G_{i}=0$. By applying the conditions (3.11) and (3.12) for $i=0,1, \ldots, \nu-1$, the theorem is established.

As a consequence of this theorem, we have the following result on maximum order.

THEOREM 2. The maximum order of multistep collocation methods given by (2.4) and (2.5) is $2 m+k-1$.

Proof. The condition $G_{i}=0$ is a nonlinear relation between $c_{1}, \ldots, c_{m} . r$ of these conditions form a set of nonlinear equations $\Gamma(\zeta)=0$ where $\Gamma: \mathbf{R}^{m} \rightarrow \mathbf{R}^{r}$. A unique solution to $\Gamma(\zeta)=0$ exists if and only if $r=m$ (see, e.g., [15, Theorem 4.2.2]).

The following theorem states that multistep collocation methods will attain maximum order only if they are in ordinate form. The argument can be found in [2, Example 12] or can be inferred from the proof of Theorem 1.

THEOREM 3. The maximum attainable order, $2 m+k-1$, for a multistep RungeKutta method is achieved only in ordinate form. The maximum attainable order is reduced by one for each $y^{(n)}$ representing approximations to derivatives.

3.3. Superconvergence, Collocation Approach. We now derive order conditions in a different form, without the use of the Alekseev-Gröbner theorem. 
As a starting point, we use an alternative expression to (3.7):

$$
u\left(t_{n+k}\right)-y\left(t_{n+k}\right)=\int_{t_{n}}^{t_{n+k}}\left(u^{\prime}(\tau)-f(\tau, y(\tau))\right) d \tau .
$$

Switching to $s$-variables, $s=\left(t-t_{n+k-1}\right) / h$, and letting $t_{n+k-1}=0, h=1$, we set

$$
\phi(s)=u^{\prime}(s)-f(s, y(s))
$$

and so

$$
\psi(s) \equiv u(s)-y(s)=\int_{0}^{s} \phi(\tau) d \tau
$$

Order $p$ then corresponds to $\psi(1)=0$ for polynomials $\psi(s)$ up to degree $p$, or the error of interpolating $y(s)$ by $u(s)$ is zero for polynomials up to degree $p$.

From the construction of multistep collocation methods we have the following interpolation conditions:

$$
\begin{array}{ll}
u(-r)=y(-r), & r=0, \ldots, k-1 ; \\
u^{\prime}\left(c_{j}\right)=y^{\prime}\left(c_{j}\right), & j=1, \ldots, m ;
\end{array}
$$

hence, for the analysis, we can choose

$$
\begin{aligned}
& \psi(s)=\pi(s) \cdot R(s), \\
& \pi(s)=\prod_{i=-1}^{k-1}(s+i), \quad R(s) \text { a polynomial. }
\end{aligned}
$$

The collocation condition then gives the order condition

$$
\left.\frac{d}{d s}[\pi(s) R(s)]\right|_{s=c_{j}}=0, \quad j=1, \ldots, m .
$$

Therefore, we have

THEOREM 4. The multistep collocation method has order $m+k-1+\nu$ if $(3.16)$ is satisfied for polynomials $R(s)$ of degree $0, \ldots, \nu-1$.

From now on we will concentrate on finding algebraic conditions on the collocation points such that the multistep collocation method has maximum attainable order $2 m+k-1$. This family of methods is related to multistep Gaussian quadrature discussed, e.g., in Krylov [7, Chapter 16]. The values of the collocation points for these $(2 m+k-1)$-order methods are therefore called Gaussian points for multistep collocation.

In order to study them, we use quadrature theory and construct the interpolation formula

$$
u(s)=\sum_{j=0}^{k-1} \alpha_{j} y(-j)+\sum_{j=1}^{m} \beta_{j} y^{\prime}\left(c_{j}\right)
$$

which follows naturally from (3.14); the $\alpha_{j}$ and $\beta_{j}$ are unknown. Order $2 m+k-1$ is equivalent to the conditions

$$
\sum_{j=0}^{k-1} \alpha_{j}(-j)^{k}+\sum_{j=1}^{m} \beta_{j} k c_{j}^{k-1}=s^{k}, \quad k=0, \ldots, 2 m+k-1 .
$$


This is an overdetermined set of equations for $\alpha_{j}$ and $\beta_{j}$, and the problem can be studied by comparing it to a related interpolation problem as done in Krylov [7, Chapter 16].

We extend the interpolation in (3.17) to include $y$-values also at $c_{j}, j=1, \ldots, m$, and we obtain the formula

$$
u(s)=\sum_{j=0}^{k-1} \alpha_{0}^{\prime} y(-j)+\sum_{j=1}^{m} \gamma_{j} y\left(c_{j}\right)+\sum_{j=1}^{m} \beta_{j}^{\prime} y^{\prime}\left(c_{j}\right)
$$

Order $2 m+k-1$ now gives

$$
\sum_{j=0}^{k-1} \alpha_{0}^{\prime}(-j)^{k}+\sum_{j=1}^{m} \gamma_{j} c_{j}^{k}+\sum_{j=1}^{m} \beta_{0}^{\prime} k c_{j}^{k-1}=s^{k}, \quad k=0, \ldots, 2 m+k-1 .
$$

These are $2 m+k$ equations for $2 m+k$ parameters. The problem can be uniquely solved provided

$$
c_{j} \neq-r, \quad j=1, \ldots, m, r=0, \ldots, k-1 .
$$

The relationship between (3.18) and (3.20) is given by the following two propositions.

PROPOSITION 5. Suppose (3.18) has a solution. Then this solution is unique, and the unknowns in (3.20) satisfy the following relations:

$$
\begin{aligned}
\alpha_{j}^{\prime} & =\alpha_{j}, & & j=0, \ldots, k-1 ; \\
\gamma_{j} & =0, & & j=1, \ldots, m \\
\beta_{j}^{\prime} & =\beta_{j}, & & j=1, \ldots, m .
\end{aligned}
$$

Proof. Obvious.

PROPOSITION 6. If the numbers $\alpha_{j}^{\prime}, \gamma_{j}$ and $\beta_{j}^{\prime}$ are a solution of (3.20) and if $\gamma_{j}=0, j=1, \ldots, m$, then the system (3.18) has a solution, and

$$
\begin{aligned}
\alpha_{j} & =\alpha_{j}^{\prime}, & & j=0, \ldots, k-1 ; \\
\beta_{j} & =\beta_{j}^{\prime}, & & j=1, \ldots, m .
\end{aligned}
$$

Proof. Obvious.

By Hermite's interpolation formula, we can write (3.19) as

$$
\begin{aligned}
u(s)= & \sum_{j=0}^{k-1} \frac{A(s)}{(s+j) A^{\prime}(-j)} y(-j)+\sum_{j=1}^{m} \frac{A_{j}(s)}{A_{j}\left(c_{j}\right)}\left[1-\left(s-c_{j}\right) \frac{A_{j}^{\prime}\left(c_{j}\right)}{A_{j}\left(c_{j}\right)}\right] y\left(c_{j}\right) \\
& +\sum_{j=1}^{m} \frac{A_{j}(s)}{A_{j}\left(c_{j}\right)}\left(s-c_{j}\right) y^{\prime}\left(c_{j}\right)
\end{aligned}
$$

where

$$
A(s)=\tilde{\pi}(s) M(s), \quad \tilde{\pi}(s)=\prod_{j=0}^{k-1}(s+j) \quad A_{j}(s)=\frac{A(s)}{\left(s-c_{j}\right)^{2}} .
$$

Now (3.18) is solvable if $\gamma_{j}=0, j=1, \ldots, m$, or

$$
\frac{A_{j}(s)}{A_{j}\left(c_{j}\right)}\left[1-\left(s-c_{j}\right) \frac{A_{j}^{\prime}\left(c_{j}\right)}{A_{j}\left(c_{j}\right)}\right]=0, \quad j=1, \ldots, m .
$$


Since $A_{j}(s) / A_{j}\left(c_{j}\right) \neq 0$, the expression in brackets must be zero. Dividing by $\left(s-c_{j}\right)$ and setting $s=-1$, we get

$$
\sum_{j=-1}^{k-1} \frac{1}{c_{i}+j}+2 \sum_{\substack{j=1 \\ j \neq i}}^{m} \frac{1}{c_{i}-c_{j}}=0, \quad i=1, \ldots, m .
$$

Thus we have

THEOREM 7. The multistep collocation method has maximum attainable order $2 m+k-1$ if the conditions (3.24) are satisfied.

COROLLARY 8. The conditions (3.24) can be formulated in the following way:

$$
\begin{gathered}
\left.\frac{d}{d s}\left[\pi(s) M^{\prime}(s)\right]\right|_{s=c_{i}}=0, \quad i=1, \ldots, m ; \\
\frac{d}{d s}\left[\pi(s) M^{\prime}(s)\right]-K(s) M(s)=0, \quad K(s) \in \Pi_{k-1} .
\end{gathered}
$$

Proof. From (3.23) we have

$$
A_{j}\left(c_{j}\right)+\left(c_{j}-s\right) A_{j}^{\prime}\left(c_{j}\right)=\left.\frac{d}{d z}\left[(z-s) A_{j}(z)\right]\right|_{z=c_{j}}=0
$$

or

$$
\frac{d}{d z}\left[\frac{(z-s) A(z)}{\left(z-c_{j}\right)^{2}}\right]_{z=c_{j}}=0 .
$$

Now $\left.(z-s) A(z)\right|_{s=1}=\pi(z) M^{2}(z)$, so we have

$$
\left.\frac{d}{d z}\left[\frac{\pi(z) M^{2}(z)}{\left(z-c_{j}\right)^{2}}\right]\right|_{z=c_{j}}=0
$$

or, equivalently,

$$
M^{\prime}\left(c_{j}\right)\left\{\frac{d}{d z}\left[\pi(z) M^{\prime}(z)\right]\right\}_{z=c_{j}}=0 .
$$

Since $M^{\prime}\left(c_{j}\right) \neq 0$, we have (3.25), and (3.26) follows immediately.

Example 9 . The case $m=2$. We can obtain the result directly from Theorem 4 and by setting

$$
\begin{gathered}
R_{l}(s)=\sum_{i=1}^{2} \alpha_{i} r_{i}(s) A_{l}(s), \\
r_{1}(s)=1, \quad r_{2}(s)=\left(s-c_{1}\right), \\
A_{0}(s)=1, \quad A_{1}(s)=\left(s-c_{1}\right), \quad \Delta_{12}=c_{1}-c_{2} .
\end{gathered}
$$

Then (3.16) gives the conditions for $\alpha_{i} \neq 0$ :

$$
\begin{gathered}
\operatorname{det}\left[\begin{array}{cc}
f_{2} & 1 \\
f_{2} & 1-\Delta_{12} f_{2}
\end{array}\right]=0 \\
\operatorname{det}\left[\begin{array}{cc}
1 & 0 \\
1-f_{2} \Delta_{12} & 2 \Delta_{12}-f_{2} \Delta_{12}^{2}
\end{array}\right]=0,
\end{gathered}
$$


where $f_{i}=\pi^{\prime}\left(c_{i}\right) / \pi\left(c_{i}\right), i=1, \ldots, m$. The last determinant gives $f_{2}=2 / \Delta_{12}$, and inserting it in the first determinant yields $f_{2}=-2 / \Delta_{12}$. Thus, the conditions for order $2 m+k-1=k+3$ are

$$
c_{1}=c_{2}+2 \frac{\pi\left(c_{2}\right)}{\pi^{\prime}\left(c_{2}\right)}, \quad c_{2}=c_{1}+2 \frac{\pi\left(c_{1}\right)}{\pi^{\prime}\left(c_{1}\right)} .
$$

In order to show that a $k(k+1) / 2$ solution set exists, we set

$$
f(x)=x+2 \frac{\pi(x)}{\pi^{\prime}(x)} .
$$

Then

$$
f(x)=x+2 / \sum_{j=-1}^{k-1} \frac{1}{x+j}
$$

and therefore

$$
f^{\prime}(x)=1+\left(2 \sum_{j=-1}^{k-1} \frac{1}{(x+j)^{2}}\right) /\left(\sum_{j=-1}^{k-1} \frac{1}{x+j}\right)^{2},
$$

so that $f^{\prime}(x)>1$.

$f(x)$ has vertical asymptotes at $k$ different points $\xi_{j}, j-1<\xi_{j}<j, j=$ $-(k-1), \ldots, 0,1$. But

$$
c_{1}=f\left(c_{2}\right), \quad c_{2}=f\left(c_{1}\right)
$$

and $c_{1}=c_{2}=j, j=-(k-1), \ldots, 0,1$, are solutions to (3.28). Hence, by looking at the case $k=3$ in Figure 2 below, Theorem 7 is established for $m=2$ and general $k$.

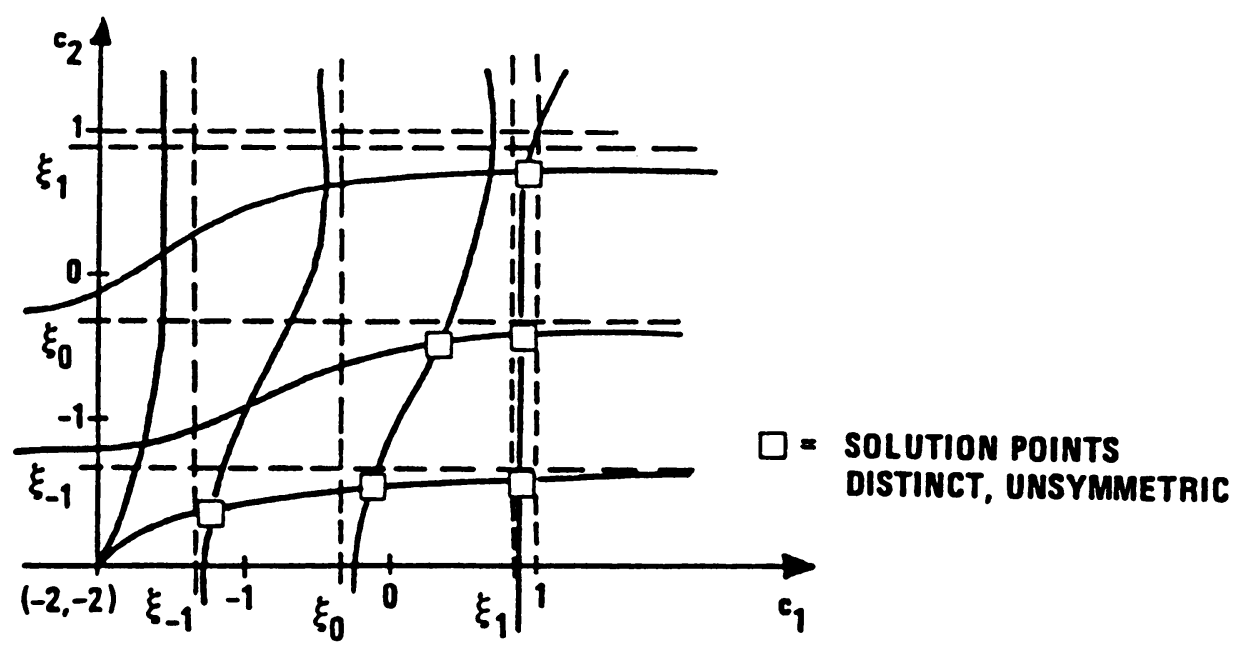

FIGURE 2

The roots of Eqs. (3.26) for $k=3$.

The general case, for $m \geq 1$ and $k \geq 1$, is more complicated, but very interesting. Krylov [7] has indicated one way of solving (3.18) and gives both the number and location of these new multistep Gaussian collocation points. Further, Munthe-Kaas 
[10] has given a different proof for the location. The result is:

THEOREM 10. There exist $\left(\begin{array}{c}m+k-1 \\ k-1\end{array}\right)$ different sets of multistep Gaussian collocation points. For each solution, all points are in $(0, k) \times \cdots \times(0, k), m$ times, and cover the number of possibilities for placing $m$ points in $k$ boxes. Each box is a unit $m$-cube.

Example $11, k=2$. As already mentioned, $k=1$ is the one-leg method case (Dahlquist [5]). In Example 7 we discussed $m=2$ in detail. Now we take a closer look at the $k=2$ case. For reasons of symmetry we transform $[0,2]$ to $[-1,1]$. With $N(x)=M(x+1)$ and $\pi(x)=x^{3}-x$, Corollary 8 shows that $N$ is given as the solution to

$$
\left(\pi(x) N^{\prime}(x)\right)^{\prime}=(\alpha x+\beta) N(x), \quad \alpha=m(m+2),
$$

where $\beta \in \mathbf{R}$ has to be such that $N \in \Pi_{m}$ is a solution. If we write $N(x)$ as

$$
N(x)=\sum_{i=0}^{m} a_{i} x^{i}
$$

and let $a=\left[a_{0}, \ldots, a_{m}\right]^{T},(3.29)$ can be formulated as an eigenvalue problem,

$$
\begin{aligned}
& (\alpha+1)\left[\begin{array}{ccc}
0 & & 0 \\
1 & . & \\
\ddots & 1 & . \\
0 & 1 & 0
\end{array}\right] a+\left[\begin{array}{cccc}
0 & & 1 . & 0 \\
-1 . & . & m^{2} \\
0 & \ddots m^{2} & 0
\end{array}\right] a=\beta a . \\
& B_{0} \quad B_{1}
\end{aligned}
$$

A simple similarity transformation shows that $A=(\alpha+1) B_{0}+B_{1}$ has $m+1$ distinct real eigenvalues. Further, by Theorem $10, N(t)$ for each $\beta$, has its zeros in $[-1,1]$.

Example 12. A list of some Gauss points for multistep collocation.

A.1. The case $m=2$. The values for $c_{1}$ and $c_{2}$ given below are solutions of the equations (3.27) now with $\pi(x)=\prod_{i=0}^{k}(x-i)$ for $k=1,2$ and 3 .

\section{TABLE 1}

Superconvergence points for $m=2$.

\begin{tabular}{|l|l|l|}
\hline$k$ & \multicolumn{1}{|c|}{$c_{2}$} & \multicolumn{1}{c|}{$c_{1}$} \\
\hline 1 & 0.7886753297 & 0.2113246703 \\
\hline 2 & 1.811992988 & 1.246307547 \\
& 1.707106495 & 0.2928930738 \\
& 0.7536913702 & 0.1880068962 \\
\hline 3 & 2.824784952 & 2.268118793 \\
& 2.733496435 & 1.350620934 \\
& 2.691981695 & 0.308018285 \\
& 1.781388803 & 1.218611204 \\
& 1.649379062 & 0.2665035178 \\
& 0.7318816461 & 0.1752150475 \\
\hline
\end{tabular}

A.2. The case $m=3$. The values for $c_{1}, c_{2}$ and $c_{3}$ in the table below are solutions of the equations (3.25) with the same definition of $\pi(x)$ as in the above case, and for $k=1,2$ and 3 . 
TABLE 2

Superconvergence points for $m=3$.

\begin{tabular}{|l|l|l|l|}
\hline$k$ & \multicolumn{1}{|c|}{$c_{1}$} & \multicolumn{1}{c|}{$c_{2}$} & \multicolumn{1}{c|}{$c_{3}$} \\
\hline 1 & \multicolumn{1}{|c|}{0.5} & \multicolumn{1}{c}{0.1127} & 0.8872985 \\
\hline 2 & 0.472138769 & 0.103442225 & 0.872652779 \\
& 0.657746108 & 0.146272877 & 1.7916885 \\
& 0.208310888 & 1.34225096 & 1.85372677 \\
& 1.52789351 & 1.12734965 & 1.89658989 \\
\hline 3 & 0.472138769 & 0.10344225 & 0.872652779 \\
& 0.630448352 & 0.136471747 & 1.75608279 \\
& 0.678545252 & 0.150853918 & 2.75017481 \\
& 0.193452664 & 1.30561654 & 1.83110796 \\
& 0.219926084 & 1.50 & 2.7800748 \\
& 0.249822439 & 2.32146216 & 2.84915055 \\
& 1.50 & 1.11644204 & 1.88356046 \\
& 1.69438365 & 1.16889076 & 2.80654825 \\
& 1.24391845 & 2.36954280 & 2.86352430 \\
& 2.54410830 & 2.13643923 & 2.90180432 \\
\hline
\end{tabular}

4. Conclusion. We have derived conditions for superconvergence for multistep collocation methods. These conditions are given in forms which are easily computable for practical values of $m$ and $k$. The analysis in this paper is done for the idea of multistep collocation. Analysis of variable stepsizes will be carried out in forthcoming papers. Another aspect to be considered is the properties of a variable coefficient version of the methods.

In [9], an error estimation technique for multistep collocation based on multistep perturbed collocation is described, and this technique has been used as a stepchanging mechanism for an experimental code described in [9]. This error estimation technique, as well as stability properties for variable stepsize, will be investigated in forthcoming papers.

Other aspects to be analyzed for multistep collocation are $C$-polynomials, singlyimplicitness and various implementation details.

Division of Mathematical Sciences

University of Trondheim

N-7034 Trondheim, Norway

1. K. Burrage, The Order Properties of Implicit Multivalue Methods for Ordinary Differential Equations, Report 176/84, Dept. of Computer Science, University of Toronto, Toronto, Canada.

2. K. BURRAGE, "High order algebraically stable multistep Runge-Kutta methods." Manuscript, 1985.

3. K. BURRAGE \& P. MOSS, "Simplifying assumptions for the order of partitioned multivalue methods," $B I T$, v. 20, 1980, pp. 452-465.

4. G. DAHLQUIST, Some Properties of Linear Multistep Methods and One-Leg Methods for Ordinary Differential Equations, Report TRITA-NA-7904, KTH, Stockholm, 1979.

5. G. DAHLQUIST, "On one-leg multistep methods," SIAM J. Numer. Anal., v. 20, 1983, pp. 1130-1138. 
6. A. Guillon \& F. L. SOulÉ, "La résolution numérique des problèmes differentiels aux conditions initiales par des méthodes de collocation," RAIRO Anal. Numér. Ser. Rouge, v. R-3 1969, pp. 17-44.

7. V. I. KRYLOV, Approximate Calculation of Integrals, Macmillan, New York, 1962.

8. I. LIE, $k$-Step Collocations with One Collocation Point and Derivative Data, FFI/NOTAT83/7109, NDRE, Kjeller, Norway, 1983.

9. I. LIE, Multistep Collocation for Stiff Systems, Ph.D. thesis, Norwegian Institute of Technology, Dept. of Numerical Mathematics, Trondheim, 1985.

10. H. MUnthe-KAAS, On the Number of Gaussian Points for Multistep Collocation, Technical report, University of Trondheim, Dept. of Numerical Mathematics, 1986.

11. S. P. NøRSETT, "Runge Kutta methods with a multiple eigenvalue only," BIT, v. 16, 1976, pp. 388-393.

12. S. P. NøRset T, Collocation and Perturbed Collocation Methods, Lecture Notes in Math., vol. 773 (G. A. Watson, ed.), Springer-Verlag, Berlin and New York, 1980.

13. S. P. NøRSETT \& G. WANNER, "The real-pole sandwich for rational approximations and oscillation equations," BIT, v. 19, 1979, pp. 79-94.

14. S. P. NØRSETt \& G. WANNER, "Perturbed collocation and Runge-Kutta methods," Numer. Math., v. 38, 1981, pp. 193-208.

15. J. M. ORTEGA \& W. C. Rheinboldt, Iterative Solution of Nonlinear Equations in Several Variables, Academic Press, New York, 1970.

16. M. ZENNARO, "One-step collocation: Uniform superconvergence, predictor-corrector methods, local error estimates," SIAM J. Numer. Anal., v. 22, 1985, pp. 1135-1152. 